\title{
Power Quality Analysis in an Industrial Electrical System by Probability Density Function
}

\author{
G. B. Gibelli ${ }^{1}$, M. Oleskovicz ${ }^{2}$ and J. C. M. Vieira ${ }^{2}$ \\ ${ }^{1}$ Department of Engineering of Energy - FAEN \\ Federal University of Grande Dourados - UFGD \\ Dourados - MS, Brazil \\ Phone number: 55673410 2160, e-mail: gersongibelli@ufgd.edu.br \\ ${ }^{2}$ Department of Electrical and Computer Engineering \\ University of São Paulo - USP/EESC \\ São Carlos - SP, Brazil \\ Phone number: 55163373 8142, e-mail: olesk@sc.usp.br, jcarlos@sc.usp.br
}

\begin{abstract}
This research aimed to analyze the Power Quality (PQ) in an Industrial Electrical System (IES) through event classification by applying the Probability Density Function (PDF). The aim is to obtain PQ indicators that enable an informative action (warning sign) for the protection devices associated through the classification of disturbances conducted by PDF. The methodology and results are based on the modeling and simulation of a real IES containing Three Phase Induction Motors (TPIM), using the DIgSILENT PowerFactory software. The modeling was performed to generate representative situations of IES operation featuring short duration voltage variations. The classification of the situations by the proposed methodology could be initially checked by observing this disturbance. Satisfactory results attest to the potential of the approach developed until now.
\end{abstract}

\section{Key words}

Power Quality, Industrial Electrical System, Probability Density Function, Three Phase Induction Motor.

\section{Introduction}

Especially in the last 15 years, there has been a growing concern among experts and researchers because of the large amount of sensitive equipment and processes affected by problems associated with the lack of a desired PQ (Power Quality). This concern arises mainly from the growing presence of non-linear loads from the new equipment installed in the Electrical Power System (EPS), which can generate and be sensitive to various problems, causing damage to final consumers of electricity [1]. For the industrial sector in particular, the increased efficiency in the use of electricity has resulted in the improvement and integration of automated processes, which in turn, may be sensitive to changes in voltage variations, and or cause changes in voltage supply in a certain Point of Common Coupling (PCC). This scenario denotes the potential economic impact on energy companies and end consumers (industrial) according to the different associated costs and resulting from problems occurred due to the lack of PQ.

It is worth mentioning that there are, in the literature, researches that apply strategies for the detection, location and classification of disturbances in the PQ area. In [2], the authors present a review of techniques and methodologies developed for PQ analysis in transmission and distribution systems, seeking to better classify the observed power disturbances. According to the proposed revision, the Wavelet Transform (WT), Transform-S (TS), Kalman Filter (KF), filter banks, and sometimes, the combination of two of them are techniques usually applied for the estimation of PQ indicators. As for the classification of events, the trend is to use algorithms based on Artificial Intelligence (AI) and Support Vector Machine (SVM), where the main problem for their application is the computational effort required and the large amount of data that should be available and analyzed.

The authors in [3] present techniques for automated analysis of various types of data collected in an EPS, emphasizing the need for an intelligent analyzer that is able to classify, identify the types and causes, and locate a short circuit, estimating all involved parameters automatically. This way, the techniques of Fourier Transform (FT), WT or TS are employed to extract the distinct characteristics of the signs under analysis and subsequently use smart tools like Fuzzy System (FS), Artificial Neural Network (ANN) or Neuro-Fuzzy for decision making of the classification of disturbances.

In the above context, from the practice, the analyzers and multimeters for PQ monitoring are embedded in a structure in IES (Industrial Electrical System) only to record, analyze and store data when an event is detected. On the other hand, the Intelligent Electronic Devices 
(IEDs) are included in IES to protect the loads or parts of the IES. As for the loads, in this specific case, the IEDs are applied to protect the Three Phase Induction Motors (TPIM), after the detection of an event that could damage the system [4].

In this scenario, for the analyzers and multimeters, there is no assignment of any function or immediate action task on the load (in this case, on the TPIM). This is valid for either a decision-making or an informative action for other processes that might use the information provided to predict or prevent the incident situation, or future situations, at the occurrence point.

Therefore, a method that may correlate characteristics of the signatures of the three phase voltage values, on the IES under observation and hence on the TPIM is highly desirable. It is believed that the result of the correlation of these signatures allow obtaining PQ indicators focused on spotting possible operation problems in TPIM, which enables it to predict and inform to IED one incident situation and perhaps damaging to the system as a whole, in a timely manner.

Thus, this research presents a study that uses the PDF (Probability Density Function), represented by histograms, as an analysis tool of PQ disturbances in an IES. The main goal of the research is to obtain PQ indicators for an informative action (warning sign) to IEDs through the classification of disturbances conducted by the PDF.

\section{Probability Density Function}

In areas related to engineering, Stochastic Process (SP) or Random Process (RP), refer to the random nature phenomena that occur in temporal or spatial manner, allowing its analysis through statistical tools. An RP is the result of a random experiment (random signal) associated to a waveform as a function of time.

Consider a random voltage signal represented by the set of its possible sample functions $X\left(t, \zeta_{1}\right), \ldots, X\left(t, \zeta_{n}\right)$ within the sample space $S$, where its sample functions depend on the variation of parameters $\zeta_{1}, \ldots, \zeta_{n}$. Thus, a random variable $X$ will be contained in the result of a random experiment by the $X(\zeta)$ number [5]. The probability distribution of a random variable $X$ can be specified with the PDF, and $f(x)$ can be used to describe the probability distribution of a random variable $X$ [6]. The probability of $X$ being between $a$ and $b$ is determined by the integral of $f(x)$ of $a$ and $b$, as shown in (1).

$$
P(a<X \leq b)=\int_{a}^{b} f(x) d x
$$

A histogram is an approximation of the PDF, where for each interval of the histogram, the bar area is equal to the frequency of the measurements on the interval and the frequency is an estimation of the probability of the measurement be in the interval. Thus, the area under $f(x)$ over any interval is equal to the real probability of the measurement being in the interval [6]-[8].

A PDF provides a simple description of the probabilities associated with a random variable. It is important to note that $f(x)$ is used to calculate an area that represents the probability of $X$ having a value in the interval $[a, b]$ as shown in Fig. 1.

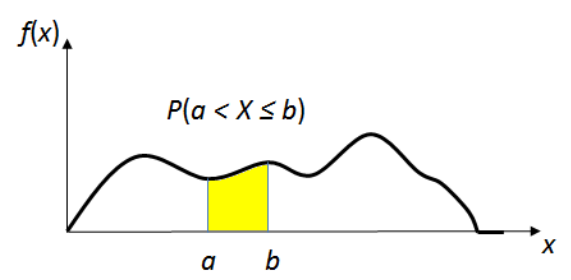

Fig. 1. Probability determined from the area under $f(x)$.

Fig. 2 illustrates a histogram in the form of bars, presenting the clusters of a set of data cells (bins). The dynamic range of data is divided into a number of cells (bins) of the same "length". The "height" of each cell is calculated by counting the number of data contained in the range [8].

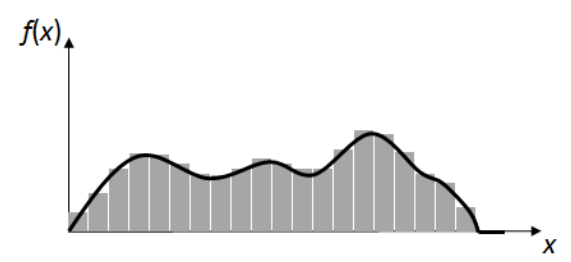

Fig. 2. Histogram in the form of bars that approximates to the PDF.

In Fig. 2, the area of each bin is equal to the interval frequency. Thus, the area under $f(x)$ over any interval is equal to the interval probability.

\section{Industrial Electrical System Analysed}

To generate representative situations of Short Duration Voltage Variations (SDVV), necessary for the analysis of the proposed algorithm, an IES was modelled considering the real data of the IES and TPIM provided by a regional industry, and using the DIgSILENT PowerFactory software [9]. Fig. 3 shows a representation of the IES used for the simulations of the loads operation and fault situations for the analysis of PQ disturbances. The situations will be analyzed through the dynamic response of the TPIM against power disturbances in the power network.

The electrical system consists of: a $138.0 \mathrm{kV}$ power substation; a $4.00 \mathrm{kV}$ three phase induction motor of $1,180 \mathrm{~kW}$; two step-down power transformers (T1 and T2) with relations $138.0 / 11.95 \mathrm{kV}$ and apparent power of 12.5 MVA; a step-down power transformer (T3) with relations $11.95 / 0.22 \mathrm{kV}$ and apparent power of 0.045 MVA; a step-down power transformer (TM) with relations $11.95 / 4.16 \mathrm{kV}$ and apparent power $1.5 \mathrm{MVA}$; and loads characterized by a unity power factor and active power of $0.04 \mathrm{MW}$ and $10 \mathrm{MW}$. It should be noted that the T1 and T2 transformers have delta-star connections with $17.25 \Omega$ grounding resistance, and the T3 and TM transformers present delta-star connections with neutral solidly grounded. The TPIM was modelled by using the asynchronous machine model of the DIgSILENT PowerFactory software [9], whereby, various electrical and mechanical parameters were 
employed. It is worth noting that the data entered in the model were taken from real motor tests in the field.

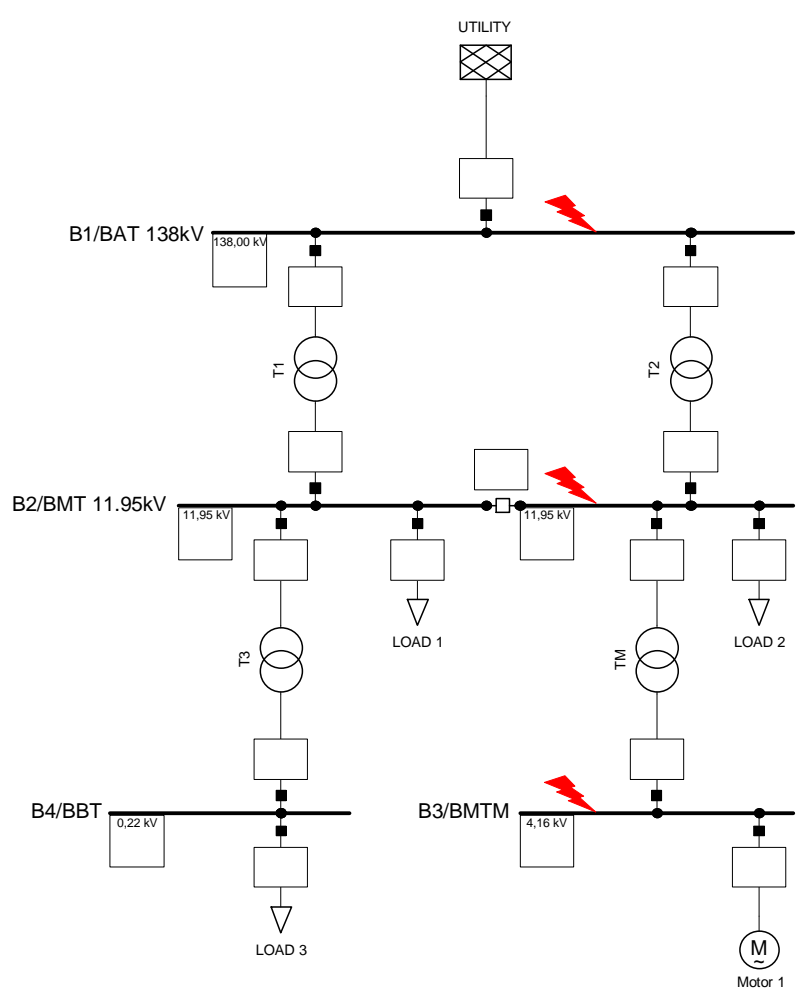

Fig. 3. Single line diagram of the IES.

Table I shows the input parameters relating to the TPIM.

Table I. - Parameters of Motor 1.

\begin{tabular}{cc}
\hline & Motor 1 \\
\hline Nominal Voltage & $4.00(\mathrm{kV})$ \\
Nominal Power & $1,180(\mathrm{~kW})$ \\
Power Factor & 0.87 \\
Efficiency & $87.4(\%)$ \\
Nominal Frequency & $60(\mathrm{~Hz})$ \\
Speed & $1,789(\mathrm{rpm})$ \\
Pole Pairs & 2 \\
Connection & $\mathrm{Y}$ \\
Locked Rotor Current & 6.70 (p.u.) \\
Locked Rotor Torque & 1.25 (p.u.) \\
Starting Torque & 2.42 (p.u.) \\
Stator Resistance & 0.105 (p.u.) \\
Stator Reactance & 0.02 (p.u.) \\
Magnetization Reactance & 3.15 (p.u.) \\
Rotor Resistance & 0.007 (p.u.) \\
Rotor Reactance & 0.421 (p.u.) \\
Rotor Type & Double Cage \\
\hline
\end{tabular}

The simulation of loads 1, 2 and 3 connected to bus B2/ BMT and B4/BBT comprises the modeling of the general load module available in the library of DIgSILENT PowerFactory software, where the provided active power is considered an input parameter [9].

Representative operating conditions that characterized interruptions, sags and swells were generated on this system, which allowed the characterization of the desired phenomena as established by the IEEE Standard 11591995 [10].
Each characterized situation considered a sampling frequency of $15,360 \mathrm{~Hz}$ (256 samples per cycle of the signals under analysis), based on the IES fundamental operating frequency of $60 \mathrm{~Hz}$.

The following 10 different faults were simulated to generate a representative database: three-phase shortcircuits; involving phase $A$ and ground (phase $A$-ground); phase $B$-ground; phase $C$-ground; phases $A$ and $B$ (phases $A B$ ); phases $B C$; phases $C A$; phases $A B$-ground; phases $B C$-ground; and phases $C A$-ground for each of the IES buses, considering the angle of fault incidence of $0^{\circ}$, fault resistance of $0 \Omega$ and duration of the fault $\left(t_{d}\right)$ of 30 cycles $(500 \mathrm{~ms})$. Measurements were performed on the B3/BMTM bus of Motor 1 for all cases of applied faults. Each of the situations contains 2 s duration that include the time on a steady-state (pre-fault), the short circuit application period (fault IES) and system restoration (post-fault).

\section{Methodology}

The methodology is based on the recording and analysis of the short circuits applied on the IES resulting in SDVV on bus B3/BMTM of Motor 1.

The initialization of the methodology consists of simulations with high sampling rate $(1 \mathrm{MHz})$ using DIgSILENT PowerFactory software in order to accurately represent the analog signals of voltage and current found in the grid.

The acquisition and conditioning of the voltage signals and sinusoidal three phase currents (instantaneous) are performed by transducers allocated close to the TPIM, using a $60 \mathrm{~Hz}$ one cycle window, at a rate of 256 samples per cycle. The displacement of the window on the signals considers $1 / 2$ step of a cycle. For this research, a data window of a cycle is taken from the moment of the short circuit application at $0.2 \mathrm{~s}$ in the IES bus.

This post-fault window will contain the three phase voltages recorded at the B3/BMTM bus of Motor 1 under analysis, as illustrated in Fig. 4.

The situation illustrated in Fig. 4 is due to the application of a phase-to-phase fault in the B1/BAT bus involving phases $A$ and $B$ of the IES under analysis.

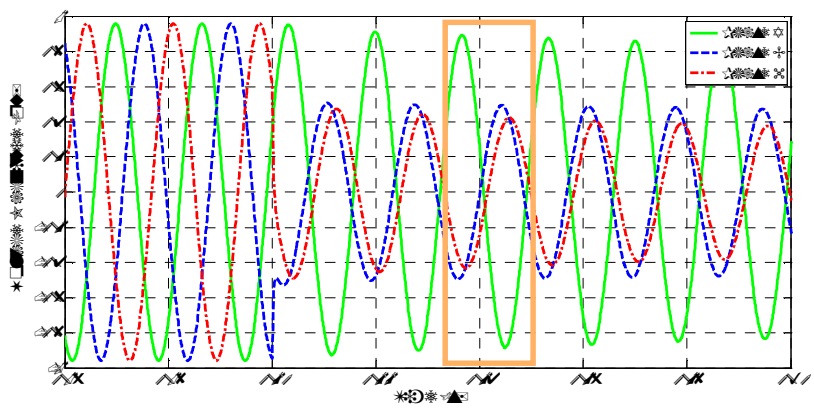

Fig. 4. Window of one cycle as an example taken from the applied fault.

Fig. 5 shows the graph of the three phase voltages containing a data window of a cycle with 256 pre-fault samples recorded on the B3/BMTM bus of Motor 1 regarding the phase-to-phase fault applied to the B1/BAT bus. 


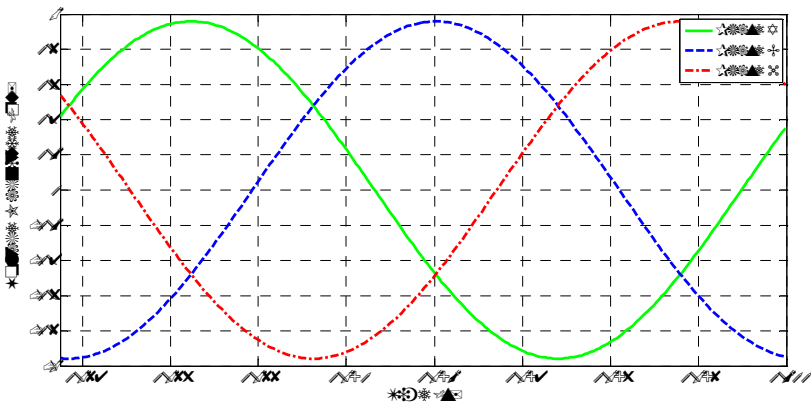

Fig. 5. Windowed three phase signal of a pre-fault cycle.

Fig. 6 shows the pre-fault histogram of phase A. After collecting the post-fault window of 1 cycle with $1 / 2$ cycle step of three phase signals under consideration, the events are classified through the analysis of the histograms approaching the PDF, and the classification of each event will be through characteristic values for each type of fault applied to the IES.

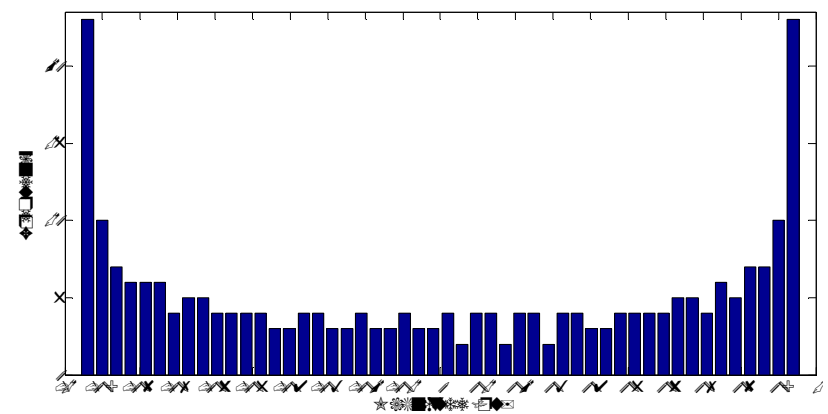

Fig. 6. Histogram of phase $A$ of the pre-fault signal B3/BMTM - M1

The histogram that represents the ratio of the frequency (occurrence number) versus the magnitude of the signal under analysis in the B3/BMTM bus in Motor 1, enables the classification of the disturbance by observing the larger number of samples (occurrences) found in maximum magnitudes of the signal in question, as shown in Fig. 7.

After execution and analysis of various simulations, an algorithm was developed through MATLAB software, which allows the analysis of the maximum magnitude values contained in the histogram bins for each voltage phase under analysis.

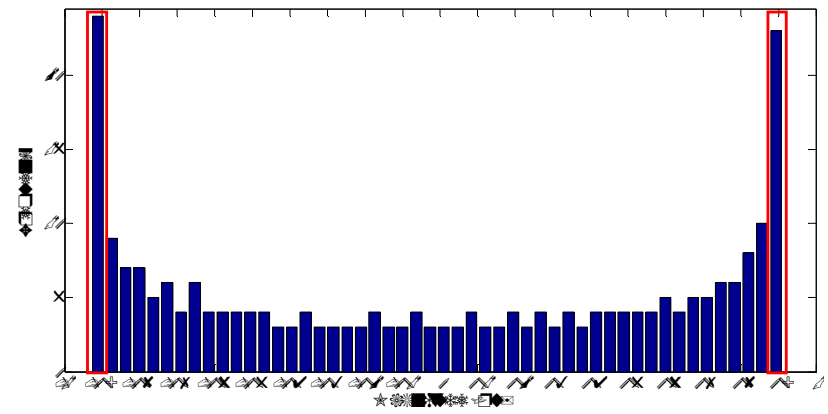

Fig. 7. Histogram reflecting the frequency (occurrence number) versus the magnitude of the signal under analysis. Phase A - Post-fault - B3/BMTM - M1.

Thus, the classification of each event associated with SDVV in the IES under analysis, was developed according to the flowchart of Fig. 8.

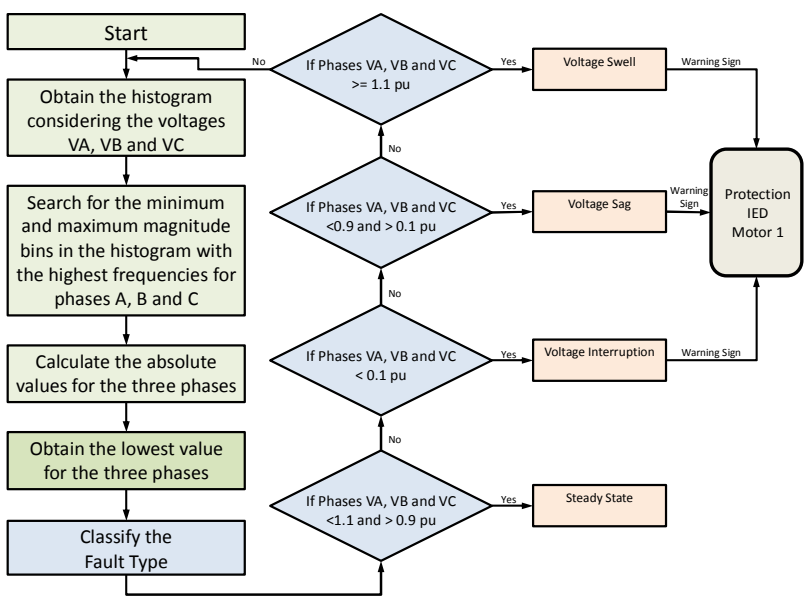

Fig. 8. Flowchart of the algorithm to classify the type of occurring event.

As a first step, the algorithm performs simultaneously the histogram calculation by the values of the ratio between the frequency (number of occurrences) and the magnitude of the signal under analysis at bus B3/BMTM on Motor 1, distributed into 50 bins (bars) for the threephases.

Further, the algorithm searches for the minimum and maximum magnitude values contained in the bins with the largest number of occurrences.

Thus, after the minimum and maximum values are found, the absolute value of the amplitude is then calculated.

With the absolute values of minimum and maximum magnitude, the algorithm seeks their lowest values and then moves to the event classification step.

Classification is performed by comparing the lowest amplitude value found to the threshold set in Fig. 9 [10].

After performing the classification, the algorithm sends a warning sign to the alarm relays of the IED.

The classification of events is based on a range of values as shown in Fig. 9. It is possible to classify the type of disturbance faced by Motor 1 from the analysis of each phase of the system simultaneously considering only one cycle of the faulty signal.

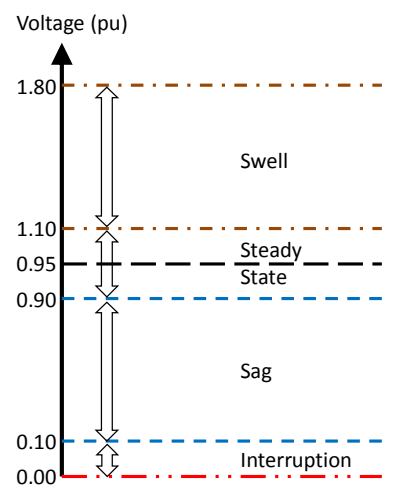

Fig. 9. Range of values considered to associate the events to SDVV (p.u.) [13].

For future work, it is intended to apply the PDF with other statistical tools that can correlate the three-phase 
signals of voltages registered with indicators that allow the prediction or forecast of the disturbance and the TPIM performance and then alert the protection IED indicating the bus, the severity and the development (evolution) of the faced disturbance.

\section{Results}

This section presents the results obtained from the analysis tool for classifying PQ disturbances in the IES. The dynamic response of Motor 1, simulated in adverse operational conditions of the IES, enabled the synthetization of the results in several characteristic patterns for each applied short circuit.

\section{A. Analysis of voltages in phases $A, B$ and $C$ resulting} from a three phase fault involving phases " $A B C$ " on the bus B1/BAT $138 \mathrm{kV}$

Fig. 10 shows a cycle of the post-fault voltage signal with window displacement of $1 / 2$ step cycle regarding phases $A$, $B$ and $C$ monitored on bus B3/BMTM in Motor 1 for a three phase short circuit applied to bus B1/BAT $138 \mathrm{kV}$.

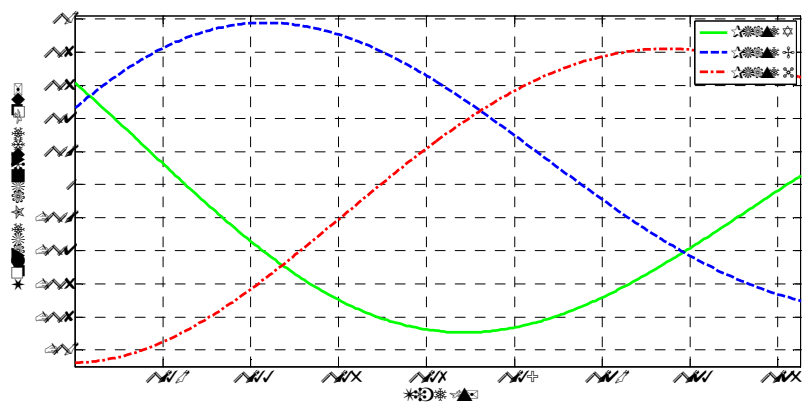

Fig. 10. A cycle of the post-fault voltage signal regarding phases $A, B$ and $C$ monitored on bus B3/BMTM - M1 to a threephase short circuit applied to B1/BAT $138 \mathrm{kV}$.

Upon such situation applied, it is possible to sort the type of disturbance through the analysis of the phase histogram. Fig. 11 shows the histogram for the frequencies of occurrence of magnitude of the voltage signal of phase $A$, where the bin on the horizontal axis shows the maximum signal magnitude of 0.08 p.u..

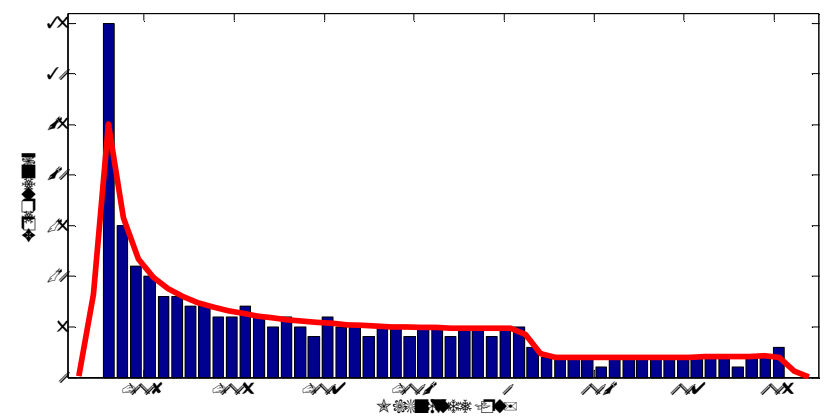

Fig. 11. Histogram graph and PDF for phase $A$ monitored on bus B3/BMTM - M1.

Figs. 12 and 13 show the histogram for the frequencies of magnitude occurrence of the voltage signal for phases $B$ and $C$, respectively. It is noted that the bin in the horizontal axis shows the maximum magnitude of the signal at 0.10 p.u. for phase $B$ and 0.08 p.u. for phase $C$.

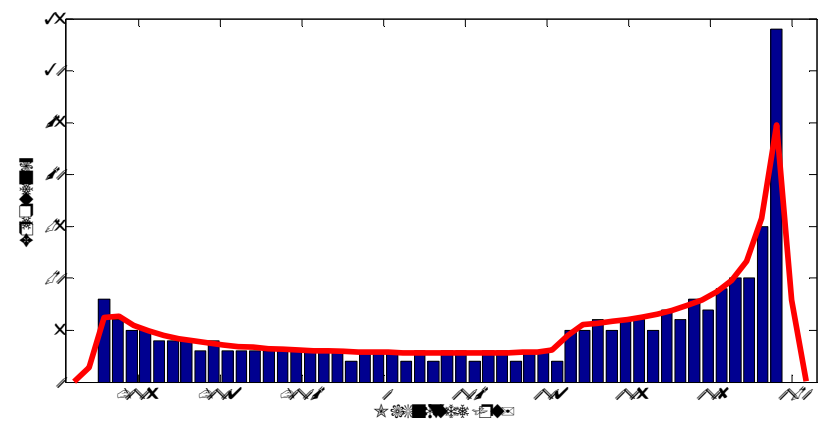

Fig. 12. Histogram Graph and PDF for phase $B$ monitored on bus B3/BMTM - M1.

Thus, the smallest value for each phase of the voltage signal was obtained from the algorithm, wherein phase $A$ presented 0.061 p.u., phase $B 0.068$ p.u. and phase $C$ 0.080 p.u.. Respecting the range of values considered to associate the events to SDVV (Fig. 9), the disturbance was rated as a voltage interruption.

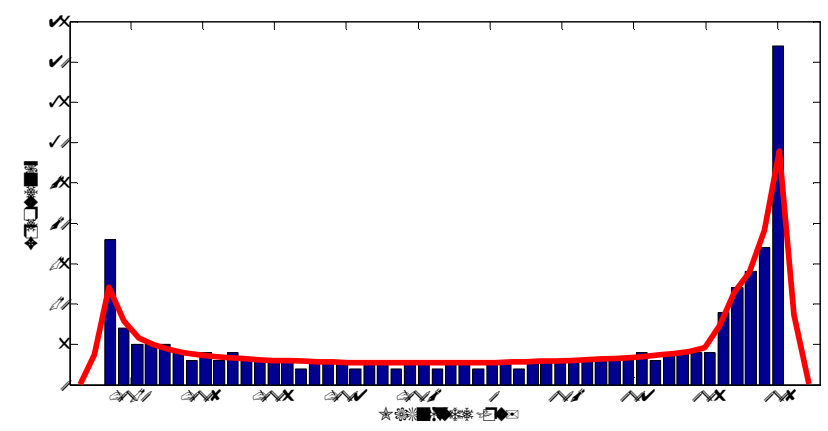

Fig. 13. Histogram graph and PDF for phase $C$ monitored on bus B3/BMTM - M1.

B. Analysis of voltages in phases $A, B$ and $C$ due to $a$ fault involving phases " $C$ " and " $A$ " on bus B2/BMT1 $11.95 \mathrm{kV}$

Fig. 14 shows the voltage post-fault signal windowed (1 cycle) at $1 / 2$ cycle step related to phases $A, B$ and $C$ monitored on bus B3/BMTM in Motor 1 , for a fault situation involving phases $C$ and $A$, applied on bus B2/BMT1 $11.95 \mathrm{kV}$.

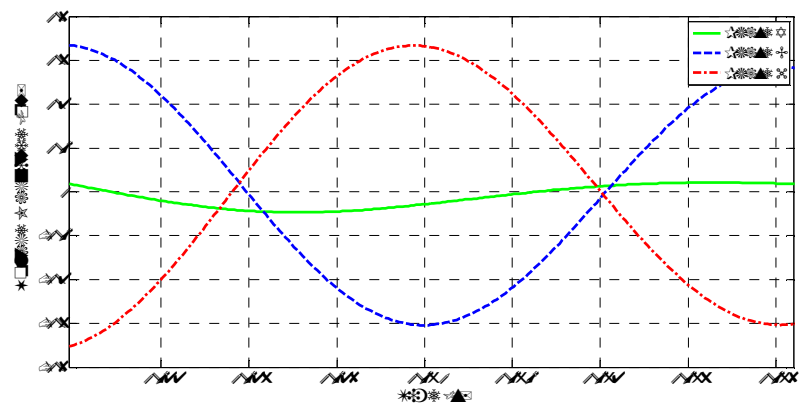

Fig. 14. Windowed signal to a phase-to-phase fault situation involving phases $C$ and $A$ monitored in B3/BMTM - M1 and applied on the bus B2/BMT1 $11.95 \mathrm{kV}$. 
Upon such situation applied, it is possible to sort the type of disturbance through the analysis of the phase histogram. Fig. 15 shows the histogram for the frequencies of occurrence of the voltage signal magnitude of phase $A$. It is noted that the bin has a maximum magnitude of the signal close to 0.04 p.u..

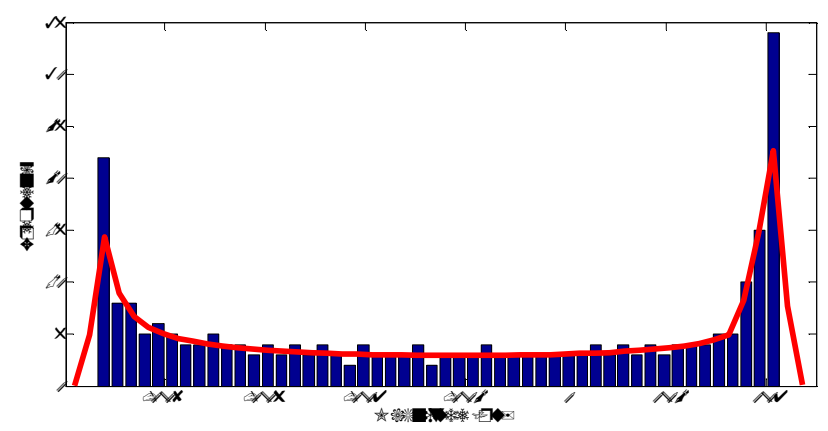

Fig. 15. Histogram graph and PDF of phase $A$ monitored on bus B3/BMTM - M1

The histogram of Figs. 16 and 17 shows that the maximum magnitude of the signals with a higher frequency are close to 0.60 p.u. for voltage signals of phases $B$ and $C$.

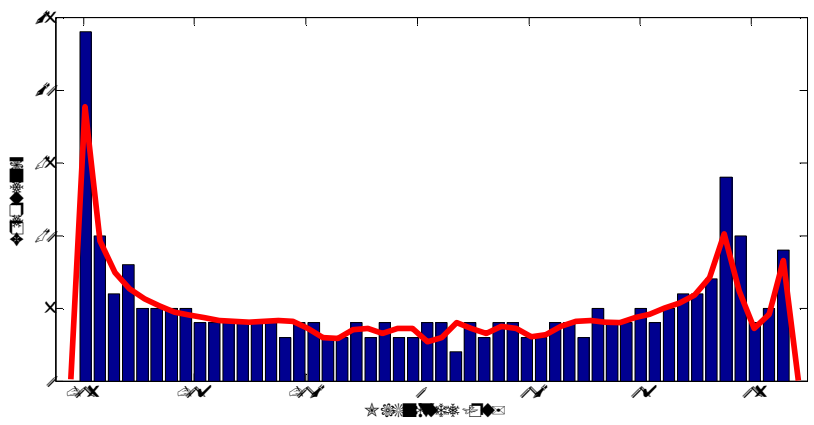

Fig. 16. Histogram graph and PDF of phase $B$ monitored on bus B3/BMTM - M1.

The type of disturbance was classified by analyzing the histogram graphs of phases $A, B$ and $C$; obtaining voltage values of 0.04 p.u. for phase $A$ (rated as a voltage interruption) and 0.59 p.u. for phase $B$ and 0.65 p.u. for phase $C$ (rated as a voltage sag), respectively.

For the moment, the proposed methodology was able to sort $100 \%$ of the events related to 30 standards presented and analyzed.

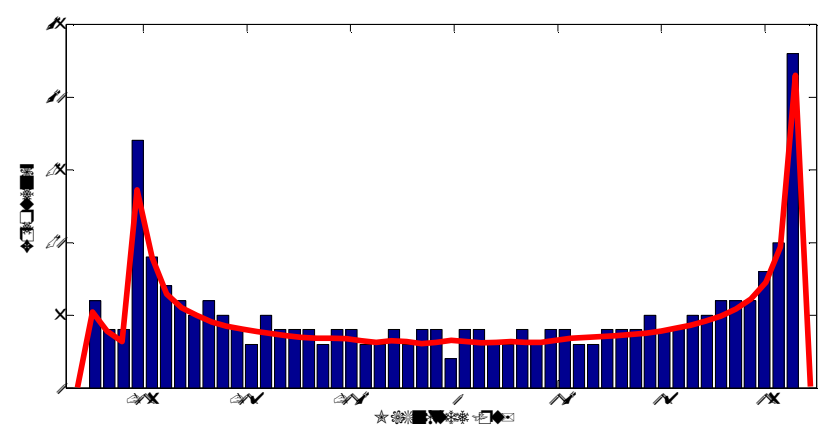

Fig. 17. Histogram graph and FDP of phase $C$ monitored on bus B3/BMTM - M1.

\section{Conclusion}

This paper presented a new stochastic-based methodology for classifying short duration voltage variations sensed by three phase induction motors in industrial plants. The main idea is to use it in a monitoring system integrated with the motors' IED so that an incident and harmful abnormal condition can be managed in a timely way to avoid damage to the machine.

The stochastic tool (PDF) used in the research is easy to be implemented and provides a fast and reliable classification of the disturbances, regardless the system bus to which the fault is applied. In addition, the results showed that the voltage variations were classified correctly in all the cases tested in the paper.

For future work, it is intended to apply the PDF with other statistical tools that can correlate the registered three phase voltage signals with indicators that allow to predict or forecast the disturbance and the TPIM performance and alert the protection IED, by indicating the bus, the severity and development (evolution) of the disturbance.

\section{Acknowledgement}

The authors would like to thank the University of São Paulo and the São Carlos School of Engineering for the infrastructure provided for the development of this work, as well as the São Paulo Research Foundation (FAPESP) and the National Council for Scientific and Technological Development $(\mathrm{CNPq})$ for the financial support provided in different parts of this research.

\section{References}

[1] R. C. Dugan, M. F. McGranaghan, S. Santoso and H. W. Beaty, Electrical Power Systems Quality, McGraw-Hill, 2012.

[2] D. G. Lieberman, R. J. T. Troncoso, R. A. O. Rios, A. G. Perez, and E. C. Yepez, "Techniques and methodologies for power quality analysis and disturbances classification in power systems: a review," IET Generation, Transmission and Distribution 5(4), 519-529, 2011.

[3] W. Fan, Y. Liao, T. Laughner, B. Rogers, G. Pitts, et al., "Intelligent data analysis for power systems," In IEEE PES General Meeting, 2012.

[4] R. Bernardes and F. Ayello, "PQMS - Power quality monitoring system: improve power systems through ieds ," in $20^{\text {th }}$ Internacional Conference on Electricity Distribution, IEEE, 2009.

[5] S. Haykin and M. Moher, Comunication Systems, Wiley, 2009.

[6] D. C. Montgomery and G. C. Runger, Applied Statistics and Probability for Motorers, LTC, 2012.

[7] J. L. Devore, Probability and Statistics for Motorering and the Sciences, Cengage Learning, 2015.

[8] Z. Gang, A. Duffy, H. Sasse and W. Lixin, "The use of Probability Density Funtions to improve the interpretation of FSV results," in IEEE International Symposium on Electromagnetic Compability (EMC), pp. 685-689, 2012.

[9] DIgSILENT PowerFactory, "Power System Analysis Software," Available: http://www.digsilent.de, 2010.

[10] IEEE 1159, "Recommended Practice for Monitoring Electric Power Quality," 1995. 